\title{
High concordance rates in the categorization of movies
}

\author{
Jake Alden Whritner \& Pascal Wallisch \\ Department of Psychology, New York University
}

\begin{abstract}
When asking people how much they liked a given movie, their agreement is low. This could be due to them disagreeing about what they saw or agreeing about what they saw but disagreeing about its valence. Film theorists, historians, and critics suggest that the categorization of films is an arduous, and frequently inconsistent task. In order to test whether this is the case empirically, we asked participants to categorize-by genres that we provided - a list of movies that was previously used in research on appraisal. We find that whereas people do not use all genres at an equal proportion, they do so consistently across observers - at a rate that is surprisingly high. We also find that this concordance in genre classification is highest if people were exposed to a similar cultural experience, such as a film studies program, whereas other factors such as biological sex or the number of movies seen did not matter. We conclude that differences in appraisal cannot be attributed to differences in categorical perception.
\end{abstract}




\section{Introduction}

People readily categorize most components of the natural world-be they people, animals, places, or things (Rosch \& Mervis, 1981). This process starts early in life (Baron, Dunham, Banaji, \& Carey, 2014) and happens for both simple (Rosch \& Mervis, 1975) and complex objects (Rosch, Mervis, Gray, Johnson, \& Boyes-Braem, 1976). It is owed to the fact that resources are increasingly scarce in higher cognition such as memory or attention. This phenomenon is well documented and can be conceived of as "bottlenecks" in cognitive processing. In attention, this manifests as early (Cherry, 1953; Broadbent, 1958) and late filters (Treisman 1964; Treisman \& Riley, 1969; Deutsch \& Deutsch, 1963), in memory it manifests as gist perception (Bartlett, 1932; Roediger \& McDermott, 1995; Kintsch, 2014). Simply put, people do not remember all or even most aspects of events or objects as they were first encountered, but store and recall only a few relevant ones. As a result, long term memory has been characterized as representing "gist"-highly simplified but meaningful caricatures of perceptual experiences (Bartlett, 1932). Remarkably, different people seem to categorize complex objects of the natural world in similar ways, even if they cannot verbalize or express the criteria by which they do so (Rosch \& Mervis, 1975). The fact that they-for the most part-do not do this idiosyncratically presumes that people have an implicitly shared understanding of what constitutes the relevant features of a given object.

However, this emerging consensus within the field of cognitive psychology is potentially at odds with prevailing views in other fields such as media, literary, and cinema studies. The categorization of movies by genre has played a prominent role in writing on film within criticism, history, and theory. For starters, many theorists and historians have considered the most common way of categorizing films - genre - to be transient at best. As Bordwell and Thompson (2012) remind us: "Because filmmakers frequently play with conventions, genres and subgenres change constantly. The broader, blanket genres such as thrillers, romances, and comedies may stay popular for decades, but a comedy from the 1920s is likely to be very different from one in the 1960s. Genres change over history. Their conventions get recast, and by mixing conventions from different genres, filmmakers create new possibilities surprisingly often" (p. 333).

This is plausible from first principles-as people watch movies, film makers learn what people want to see. At the same time, the experience of watching movies changes the discernment of the viewer. Both of these ongoing and interacting processes are likely to change genres over time. This view is also echoed in the film studies literature when Rick Altman (1984) - in his seminal article on film genres_-gives credit to the viewer when he says: "By choosing the films it would patronize, the audience revealed its preferences and its beliefs, thus inducing Hollywood studios to produce films reflecting its desires. Participation in the genre film experience thus reinforces spectator expectations and desires" (p. 9).

In addition, critics have frequently pointed out that the very notion of genre is deeply problematic. A literature review of genre theory suggests a lack of census about the general constituents of genre. For example, Steven Neale (1990) proposes that while 
individual viewers have their own idiosyncratic classifications and generic expectations, the institutional discourse - that is, the idea of the film that is circulated and promoted by production and marketing companies and the filmmakers themselves-should be of central importance for genre studies (pp. 48-52). Conversely, Bordwell and Thompson (2012) suggest that: "For viewers, genre categories are a part of their tastes. Every moviegoer likes some genres, tolerates others, and loathes still others. Fans may try to see everything in a genre they love and to learn as much as possible about their favorites" (p. 331). Others, such as Alan Williams (2009), have emphasized the need to get out of the United States when thinking of genre and take a more cross-cultural approach (p. 124). We conclude that there is little consensus within film theory as to whether genres are universal or idiosyncratic. Put differently, whether each individual viewer constructs genres in a unique fashion or whether cultural forces such as marketing and production efforts impose a stable cognitive structure on the viewer.

Regardless of which of these views is most viable, the notion that people have a multifaceted relationship with genres is plausible. Complex audio-visual stimuli like commercially produced films afford the viewer a considerable degree of freedom to construct the experience idiosyncratically. Indeed, it has been shown that the agreement about whether a movie is "good" is rather low (Wallisch, 2005; Wallisch, 2014; Wallisch \& Whritner, 2017). This raises the question whether people disagree as to the quality of the movie because they disagree about what they are watching-for instance by focusing on different aspects of the movie-and constructing the experience radically differently, or whether they agree about what the movie is, but disagree about its valence.

A potential way to answer some of these open questions comes in the form of empirical methods. As a matter of fact, this was pointed out more than 20 years ago: "[...] there has hardly been any empirical research on the ways in which real audiences might understand genre, or use this understanding in making sense of specific texts" (Buckingham, 1993, p. 137). Even though Buckingham himself conducted some empirical research on children's understanding of television genres in the UK-he showed that children start to talk about their television preferences in terms of genre starting at around age 8-this remains true today. The notion of how well people are able to categorize movies has not been tested empirically. Here, to answer this question and to fill this gap in the literature, we explicitly test how people categorize movies.

It is not our intention to engage with the ongoing debate within film studies on genre theory and criticism per se. Nor is it our goal to settle once and for all what should be considered the canonical genres or an exhaustive list of what films might comprise them. This study will not address what defines a genre for critical and theoretical use.

However, we will be in a position to address the question of whether people categorize a movie in the same way or idiosyncratically. 


\section{Methods}

\section{Materials}

In this research, we used a list of 209 movies that was utilized in previous work on movie taste (Wallisch, 2005; Wallisch \& Whritner, 2017). We also created an inventory of ten categories that we thought would correspond to popular film genres and span the space of all popular movies. We also included a "miscellaneous" category for things that don't fit any of the other genres. We reasoned that usage of this category would be a good metric of whether we succeeded in covering the space of genres. Importantly, we also attributed a primary emotion, mood, or feeling that is elicited or prominently featured by the films in a given genre, see table 1 for a list of genres and emotions.

\begin{tabular}{|l|l|}
\multicolumn{1}{|c|}{ Genres } & \multicolumn{1}{c|}{ Primary emotion } \\
\hline Action & Intensity (visual) \\
\hline Art & $\begin{array}{l}\text { Aesthetic } \\
\text { Appreciation }\end{array}$ \\
\hline Comedy & Laughs \\
\hline Documentary & $\begin{array}{l}\text { Information/Propa } \\
\text { ganda }\end{array}$ \\
\hline Drama & Conflict \\
\hline Horror & Fear \\
\hline Musical/Opera & Intensity (aural) \\
\hline Romance & Love \\
\hline Sci-fi/Fantasy & Awe \\
\hline Thriller & Suspense \\
\hline Misc. & Anything else \\
\hline
\end{tabular}

Table 1. This table shows the categories that were available to our participants for judging the movies in the left column and the primary emotions, moods, or feelings that we associated with it. Note that participants were instructed to use the "Misc." (Miscellaneous) category in case they concluded that a given movie does not adequately fit any of the other categories.

\section{Participants}

Research participants were 32 former or current students at New York University, ranging in age from 18 to 34 (24 white, 6 asian, 2 black). Our sample was matched in terms of biological sex (16 males, 16 females). Also, 16 of them were cinema-studies students, whereas 16 were not. All participants were naive as to the motivation and purpose of this study. 


\section{Procedure}

We instructed participants to assign a primary and secondary genre to each movie on the list, keeping in mind the emotion, feeling, or mood outlined in table 1. Participants proceeded to fill in this information in an Excel spread sheet independently and on their own time. They were instructed to skip the movies that they had not seen. Importantly, we also instructed them to not overthink this task and simply assign primary and secondary genres to movies as best they could.

\section{Data analysis}

We calculated the concordance rate-whether participants assigned the same or different genres for each movie-using MATLAB (The MathWorks Inc., Natick, MA, USA). This rate can vary from 0 to 1 if everyone who saw the movie picks a different genre or if everyone who saw the movie picks the same genre respectively-and everything in between.

\section{Results}

\section{Do people use our categories equally when judging movies?}

Before we can assess whether people make the same judgment as to whether a given movie belongs to a particular genre or not, we first need to establish if they use the categories we provided with an equal proportion. This is important to construct the noise distribution against which we judge whether our empirical result is likely to happen by chance. For instance, if people used all categories with an equal likelihood, we could simply pick from the available categories at random. If they do not, the appropriate statistical procedure is to reshuffle the categories as they were used by the participants. Here, we test whether this is the case or not.
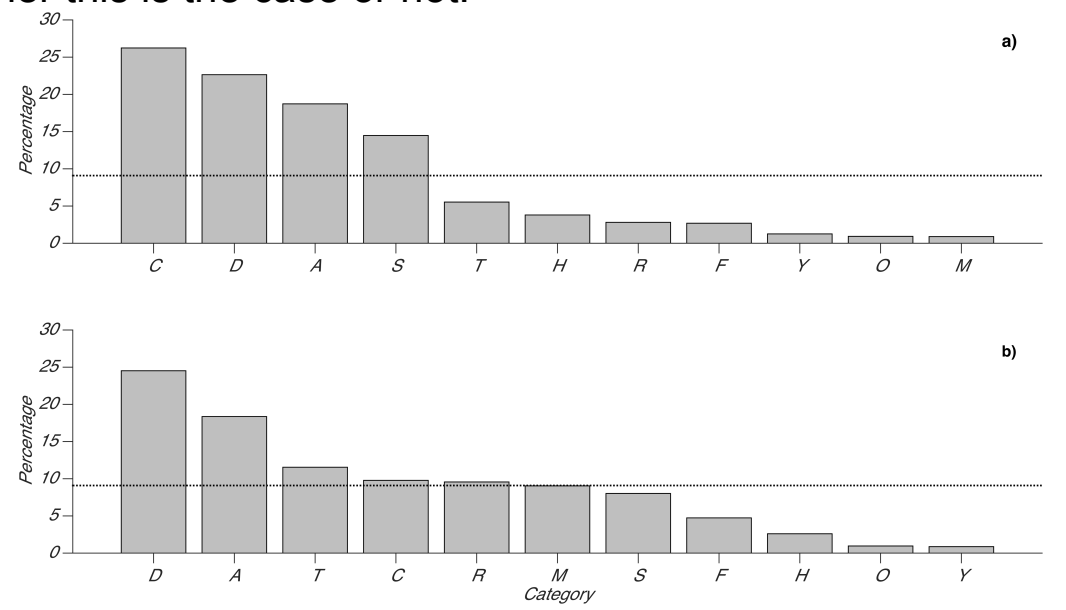

Figure 1. Category use by study participants for primary and secondary genre. a) The upper panel shows the use of primary genres within our pool of participants. On the $x$-axis are the respective categories $(C=$ Comedy, $D=$ Drama, $A=$ Action, $S=$ Sci-fi/Fantasy, $T=$ Thriller, $H=$ Horror, $\mathrm{R}=$ Romance, $\mathrm{F}=$ Art, $\mathrm{Y}=$ Documentary, $\mathrm{O}=$ Musical/Opera, $\mathrm{M}=$ Misc.), on the $\mathrm{y}$-axis is the percentage of our participants that used that particular category. Grey bars represent the empirical rate at which our participants used a given category. The dotted, black horizontal line represents the category use that we would expect if our participants were to use these categories equally. b) The lower panel depicts everything in the same way as in a), but for secondary genres. 
Figure 1 shows the category use of our study participants for primary and secondary genre. We performed a chi-squared test and found that participants do not use categories equally, neither for the primary $(x=3367, d f=10, p<1 e-300)$, nor the secondary genre $(X=1871, d f=10, p<1 e-300)$. Qualitatively, only 4 categories (Comedy, Drama, Action, and Sci-fi/Fantasy) accounted for $82 \%$ of the total, for primary genres. The use of secondary genre was a bit more diverse, it takes 6 categories-or more than half-to account for $82 \%$ of the total.

We conclude that our participants did not use the available categories equally, but we cannot tell whether this is due to the participants or the movies. We intuit that people would use some categories more frequently simply as a result of the kinds of movies we selected for the study. As we decided to use mainstream films produced in the United States, it is probable that certain categories-those that have a history of commercial success within the U.S. and Hollywood in particular-would be featured more prominently than others, such as comedies, drama or action movies. Conversely, it is also possible that participants are perceiving movies in a certain fashion (e.g. as comedies or dramas), regardless of how the filmmakers, press, and critics are branding them. Of course, these possibilities are not mutually exclusive. That said, which of these possibilities is the case is not relevant to answer our other questions and will have to be the subject of future investigation.

\section{Do people categorize movies in the same way?}

Whereas we can't address why people use these categories in this way, here we ask whether they do so consistently across observers or whether category use is highly idiosyncratic. Previous research has shown that appraisal of these movies is highly idiosyncratic (Wallisch, 2005; Wallisch \& Whritner, 2017). This leaves open the question of whether this is also true for how people perceive these movies.

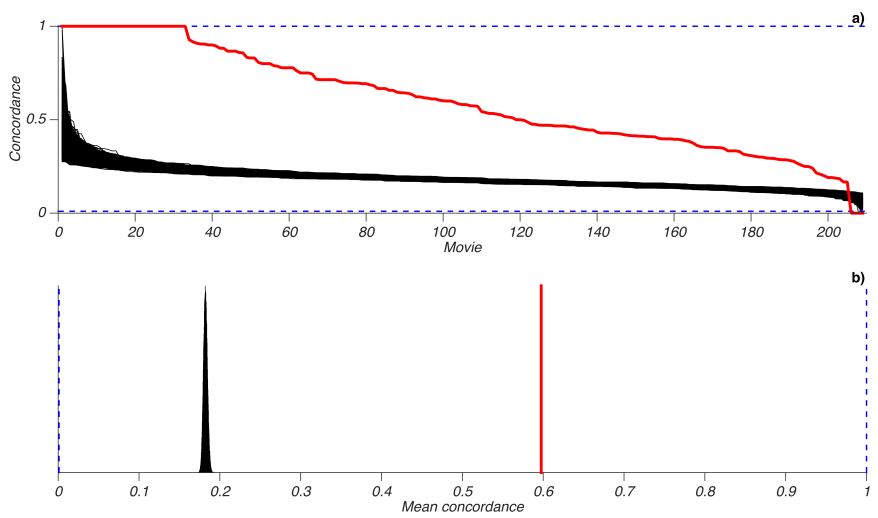

Figure 2. Concordance of category use across participants. a) The upper panel shows the empirical use of categories compared to a simulation of people using them randomly. The x-axis represents the 209 movies, sorted by concordance and the y-axis the concordance rate. The red trace depicts the empirical concordance rate as found in our sample. Each black trace represents one run of our simulation. Blue dashed lines represent the theoretically possible maximal and minimal values. b) The lower panel shows the distribution of mean concordance values (average of all movies in panel a). The $x$-axis represents the mean concordance rate and the $y$-axis the proportion of samples with a particular concordance rate. The black lines depict a histogram of all means values yielded by our simulation. The vertical red line represents the empirical mean. The vertical dashed blue lines represent the theoretical minimum/maximum. 
In order to address this question, we compared the empirical concordance rates per movie to the distribution of concordance rates yielded by randomly shuffling the categories as used by our participants (see figure 2). This accounts for the fact that people don't use categories equally (see figure 1) and have not seen all movies; thus, this is a fair comparison. In figure $2 a$, we ordered the movies by concordance rate $(1=$ all participants agreed, $0=$ all participants differed in their categorization), using information from primary genre only. The "noise distribution" in black was constructed by reshuffling 100,000 times at random. The empirical distribution of category use is much more consistent than expected by chance. Not a single of our 100,000 runs comes even close to replicating our empirical distribution. The mean concordance in our sample for primary genre was 0.598 - the highest value we saw in our simulation was 0.195 - and that was an outlier. The mean concordance of the noise distribution obtained by random shuffling was 0.182 ( $S D=0.0025, z=164.59, p<1 e-300)$. It is particularly notable that whereas some simulations can occasionally yield a perfect agreement, this drop-off is rather sharp, whereas the empirical distribution has a wide "concordance plateau" of 33 (about 1 in 7) movies with perfect agreement. This is not owed to the fact that these are movies that only a few participants have seen, which would make agreement more likely; of the 33 movies that had $100 \%$ agreement, the average number of seen was 13. Whereas it is true that disagreement becomes more likely if the numbers of people who have seen a given movie is low (all 4 movies with zero agreement have-at most-been seen by 3 people), empirically, there is no correlation between how many movies people have seen and concordance $(r=0.02, p$ $=0.74$, n.s.). On the contrary, there are quite a few movies that have been seen by everyone or almost everyone *and* have perfect or close to perfect agreement. For instance, the movies Elf (2003) and American Pie (1999) were quite popular - all but three of our participants reported on having seen them, and they all agreed on their primary designation as a Comedy, a level of agreement that is extremely unlikely to happen by chance alone. Conversely, movies like Gods and Generals (2003) have only been seen by 2 of our participants, and in the analysis above, we scored it as agreement level "zero" because the two participants disagree - one classifying it as Action whereas the other classified it as Drama. Yet, both participants agreed on the two categories involved (Action and Drama), they just disagreed about their orderprimary and secondary genre. As we are not primarily interested in order effects-and don't know how strongly people felt about the primary vs. secondary designation-we wondered whether this analysis underestimates the actual level of agreement. Thus, we performed an additional analysis, taking the information from the secondary genre into account, but discounting order. From first principles, taking the information from the secondary genre into account without taking order into account will make perfect matches less likely - everyone might agree on a primary genre (say comedy), but not on the secondary genre. In addition, if people really did agree on categories, but not on order, it should make complete non-matches also less likely. Empirically, we find that this is the case (see figure 3 ). 

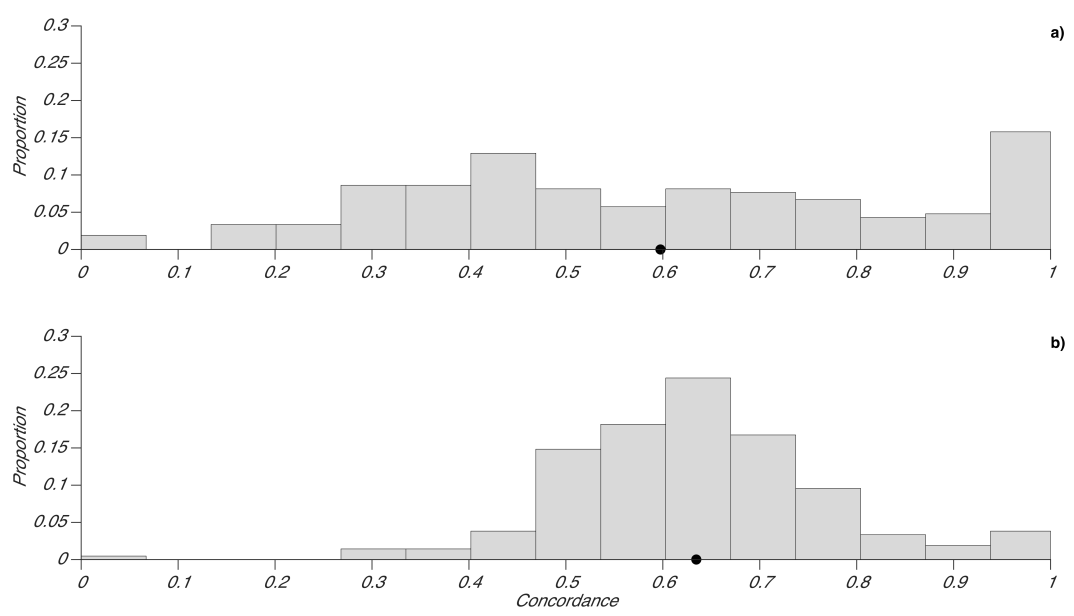

Figure 3. Comparing the concordance rates of primary category use across participants to both primary and secondary category use. a) The upper panel shows concordance rates of primary categories only. The $x$-axis represents the concordance rate and the $y$-axis represents the proportion of the movies that fall into the bin. The black dot on the x-axis represents the sample mean. b) The lower panel depicts everything in the same way but shows the concordance rates of both primary and secondary category use.

If anything - doing the analysis this way strengthened our conclusions. Now, only a single movie-Chain Reaction (1996) scores a concordance of below 0.25 . This is likely owed to the fact that it is a rather obscure movie, only 2 of our participants reported on it, and they happen to disagree on all counts. Yet, even when taking secondary genre into account, we find perfect matches for 7 of the 209 movies. As we predicted, taking the information from secondary genre into account moved the overall level of agreement into the middle, away from extremes, see figure $3 \mathrm{~b}$. The mean concordance when taking only primary genre into account is 0.598 , and it is 0.635 when taking both genres into account, a difference which is not statistically reliable $(\mathrm{t}(416)=$ $1.79, p=0.07$, n.s.).

\section{Are there effects that moderate category use?}

We just established that people mostly use categories in the same way, but the agreement among observers is not perfect. Here, we wondered whether there are systematic group factors that could account for these differences. First we asked whether biological sex plays a role and whether there are differences in how male and female participants used these categories.

It is conceivable that one could have two groups that both have perfect concordance rates amongst themselves, but that drop much lower once one averages across groups. As we did this for our overall analysis (see figure 2), we need to establish whether there are such subgroups. In terms of biological sex, that does not seem to be the case. The average concordance rate among males was 0.632 for primary genre use and 0.643 when using both categories, compared to 0.578 and 0.622 for females, respectively. The differences between these comparisons are not significant- $t(388)=1.54, p=$ 0.123 , ns. and $\mathrm{t}(388)=1.02, \mathrm{p}=0.307, \mathrm{df}=388$, n.s. respectively. Note that for this comparison - as for all others - we have to adjust for the number of movies that were seen by less than 2 people. If this is the case, we cannot compare how someone else categorized a given movie. Concordance is only defined for cases where at least 2 
people report on the same movie. For instance, in the case of biological sex we had to remove 6 movies that only one male had seen and 13 movies from the females. Without removing these cases, in principle, it is possible that the comparison becomes unfair, as it is done on the basis of different movies. If movies that are seen by less people differ systematically in their concordance from others, this could be problematic. However, we did not find this to be the case empirically. If one excludes all movies from consideration that are seen by less than 2 people in *either* group, the new concordance value that is based on matching primary genre is now 0.626 and 0.581 for males and females respectively. In other words, the absolute values changed hardly at all (and nonsignificantly). Remarkably, this is the case for all groups, including the difference between people who had seen a lot of movies and people who seen few. In the latter group, we had to remove 58 movies on these grounds, yet the absolute value did not change appreciably. So whereas we here report on the fair comparison between the groups (using the same movies for both), this did not matter in practice. To summarize, males and females do not seem to categorize differently, as measured by concordance rate.

We then wondered whether cultural factors, such as being exposed to a film studies program would matter. Conceptually, this would make sense, as such a program provides correlated inputs (analyzing similar movies on a curriculum) as well as providing common tools to analyze formal and stylistic elements of film (e.g., narrative structure, editing techniques).

Indeed, this seems to be the case empirically. The concordance is much stronger among people who studied film (we call them "experts" in the following) than those who did not. The concordance rate among experts was 0.625 for primary genre and 0.657 for both genres, compared to 0.476 and 0.556 for non-experts, respectively. These differences are substantial and statistically significant: $\mathrm{t}(312)=4.71, \mathrm{p}<0.001$ and $\mathrm{t}(312)=5.099, \mathrm{p}<0.001$ respectively. However, the average movie was seen by 11 people in the group of experts and only 5 people in the group of non-experts, so a possible confound could be the mere fact that the experts - being experts - have simply seen a lot of movies, instead of them sharing a joint culture of how to think about these movies. Therefore, we conducted a median-split of our sample, into people who saw more movies than half the people in the sample and those who saw less than half, regardless of expert status. The concordance rate among people who saw more movies was 0.613 for primary and 0.647 for both genres, whereas it was 0.549 and 0.612 for people who saw less movies, respectively. Importantly, these differences are not statistically significant: $\mathrm{t}(300)=1.73, \mathrm{p}=0.085$, n.s. and $\mathrm{t}(300)=1.72, \mathrm{p}=0.086$, n.s. We conclude that mere exposure to movies is not underlying the differences in concordance that we saw above, whereas learning a particular way of thinking about them is, see figure 4 for a summary of these results. 


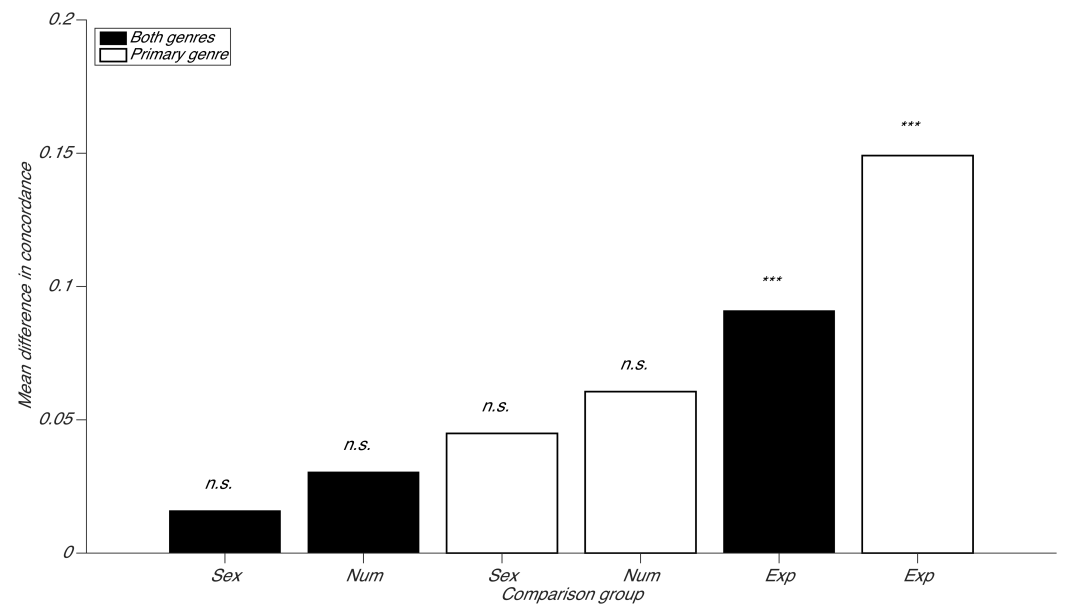

Figure 4. Moderator effects (sex, number of movies seen, expertise) on absolute mean concordance rate. Black bars depict concordance rates when taking both genres into account. White bar represents concordance rates of primary genre only. Stars indicate significance level: ${ }^{*}=p<0.05,{ }^{* *}=p<0.01,{ }^{* * *}=p<0.001$, n.s. $=$ not significant.

\section{Discussion}

We asked research participants to categorize movies they had seen in terms of primary and secondary genre. We showed that our participants did not use the genre categories available to them equally often, but that they did so much more consistently than would be expected by chance. This concordance is high and similar in absolute terms, regardless of whether we only use the designated primary genre or whether we include information from both genres. One could argue that someone who categorizes a movie as action/drama does not have zero agreement with someone who categorizes it as drama/action instead. Our study is not aimed at discerning which genre is more prominently perceived, but simply which categories are used to describe a movie and not the degree to which this is the case. Regardless, this did not affect our conclusions.

Finally, we wondered if there are any moderators that could modulate concordance rates. We considered biological sex, expertise and number of movies seen as potential factors. Of these, expertise had a significant moderating effect. This is reasonable, as a shared expertise-in this case having been a film studies student—might constitute common ground in the form of shared interests, common knowledge about movie history and ways of analyzing a film (Keysar, Barr, Balin, \& Paek, 1998). Interestingly, the concordance rate was higher among the film students. It could be argued that a priori, film students constitute a highly idiosyncratic and sophisticated subpopulation and that their agreement could be expected to be less than the naive judgments of the uninitiated. However, this is not what we find empirically. This effect could not be attributed to number of movies seen. Whereas the film students had seen significantly more movies than the non-film students, simply comparing people who had seen more movies to people who had seen less movies did not produce significant differences in concordance rates. Similarly, there were no differences in concordance rates due to biological sex. This is perhaps not surprising - males and females probably see the world in more or less the same way. 
These findings suggest that dramatic differences in the appraisal of a given movie cannot be reduced to a different perception of the movie, which was a theoretical possibility (Wallisch, 2005; Wallisch \& Whritner, 2017). For instance, the fact that one person likes a movie and another one doesn't could be due to the possibility that one person perceives it as a comedy whereas the other perceives it as a drama. However, empirically we almost never find this. For example, in the case of the movie American Pie, everyone in our sample agreed that the movie was a comedy, people simply disagreed whether it succeeded in its attempt at humor or not. This suggests a two-step process of movie appraisal. A categorical one in which there is a considerable degree of agreement between observers and a subsequent evaluative one, in which there is not.

In addition, our results are of interest to film studies due to the complicated nature of genre theory and the fact that hardly any evidence has been collected on how people categorize films empirically. Given the diverse opinions about genre within film theory and the uncertainty about whether each viewer constructs genres idiosyncratically or is influenced by cultural discourse, marketing factors, or the opinion of film critics, the high degree of concordance amongst our participants-particularly those with knowledge of genre theory - is striking. The difference between expected concordance rate by chance and our empirically observed one is not only statistically significant but truly substantial-our observed sample mean concordance is well more than a hundred standard deviations above the one expected by chance. Thus, our results indicate that despite the complicated way in which theorists describe genre use, people do not seem to have much difficulty when it comes to categorizing films and agreeing on which categories are suitable to describe a given film.

In terms of implications for cognitive psychology, these results are also somewhat surprising. Whereas people routinely categorize even complex objects effortlessly, they often do so differentially, with an idiosyncratic set of categories and their members. Movies are highly complex, yet the observed concordance rate is very high-for more than $15 \%$ of the movies we asked about, every participant agreed on the most suitable category. This raises the question as to what makes movies special. One possible explanation lies in film's ability to guide viewers' attention so effectively. Tim J. Smith, for example, has studied this phenomenon at length in his Attentional Theory of Cinematic Continuity (AToCC) (2012). He refers to this ability as "the tyranny of film" (personal communication). This possibility is supported by studies on eye movements which showed that people essentially look at the same regions on the screen for a given cut (Smith, 2012; Wang, Freeman, Merriam, Hasson, \& Heeger, 2012; Smith, 2013). As movies are essentially snippets of simulated reality (and treated by the brain as such), this opens the intriguing possibility that perception is inherently categorical.

There are several considerations that potentially limit our general conclusions. For instance, one could argue that providing people with genres to use in categorization instead of soliciting their own idiosyncratic categories was not a fair test of concordance. However, people used our genre categories spontaneously and without hesitation. Only a single participant complained that he is missing the genre "adventure" on our list. Importantly, this problem did not seem to be common, as the "miscellaneous" category 
that participants were instructed to use if none of the other categories fits was used extremely rarely. If we were missing anything crucial from our list of categories we would have seen the "Misc." category used more frequently. Also, we do not know what would have happened if participants were using completely arbitrary categories. Whereas it is possible that other categories are used in other ways, we feel confident that our categories cover the space of top-level genres nicely. This raises an issue that essentially precludes letting participants use entirely idiosyncratic categories, as the level of genre to be used is unclear. For instance, there is an arbitrary number of subgenres, e.g., comedy subdivides into romantic comedy, slapstick, black comedy and many others. Confusion about which level of genre is appropriate could mimic as an apparent disagreement about what top-level genre a given film is, even if people agree about that. Our approach avoids this problem. Our goal was to see whether informed viewers could agree on the categorization of these 209 films and we found that they overwhelmingly can.

Whereas our sample was representative of NYU's student population in terms of race and age, it might not be representative of the population at large. Put differently, some of our high concordance rates can conceivably be attributed to a restricted range in terms of age, race, socioeconomic and educational states, rendering their judgments more similar to each other than they would be otherwise. If this is the case-and if these factors matter for categorization-we would predict concordance rate to be somewhat lower in the general population. However, this is a problem shared with most psychological research (Jones, 2010).

There are several avenues of future research within this framework. One of them would be an explicit test of this two-step model of perception-namely that people can generally agree about what they are looking at, but cannot agree on whether it is good or not. Another obvious route would be to extend this work to other domains and media, e.g. music.

Similarly, it would be interesting to repeat this study with a more diverse population to see if these results generalize. We hypothesize that they will replicate qualitatively-that we will still observe surprisingly high concordance rates_but that the magnitude of this effect will be somewhat dampened. 


\section{References}

Altman, R. (1984). A semantic/syntactic approach to film genre. Cinema Journal, 23(3), 6-18. http://doi.org/10.2307/1225093

Baron, A. S., Dunham, Y., Banaji, M., \& Carey, S. (2014). Constraints on the acquisition of social category concepts. Journal of Cognition and Development, 15(2), 238268.

Bartlett, F. C. (1932). Remembering: An experimental and social study. Cambridge: Cambridge University.

Bordwell, D., \& Thompson, K. (2012). Film art: An introduction (10th ed.). New York: The McGraw-Hill Companies.

Broadbent, D. E. (1958). Perception and communication. Pergamon Press.

Buckingham, D. (1993). Children talking television: The making of television literacy. London: Falmer Press.

Cherry, E. C. (1953). Some experiments on the recognition of speech, with one and with two ears. The Journal of the acoustical society of America, 25(5), 975-979.

Jones, D. (2010). A WEIRD view of human nature skews psychologists' studies. Science (Washington), 328(5986), 1627-1627.

Keysar, B., Barr, D. J., Balin, J. A., \& Paek, T. S. (1998). Definite reference and mutual knowledge: Process models of common ground in comprehension. Journal of Memory and Language, 39, 1-20.

Kintsch, W. (2014). The Representation of Meaning in Memory (PLE: Memory). Psychology Press.

Mervis, C. B., \& Rosch, E. (1981). Categorization of natural objects. Annual review of psychology, 32(1), 89-115.

Neale, S. (1990). Questions of Genre. Screen, 31(1), 45-66. http://doi.org/10.1093/screen/31.1.45

Roediger, H. L., \& McDermott, K. B. (1995). Creating false memories: Remembering words not presented in lists. Journal of experimental psychology: Learning, Memory, and Cognition, 21(4), 803-814

Rosch, E., \& Mervis, C. B. (1975). Family resemblances: Studies in the internal structure of categories. Cognitive psychology, 7(4), 573-605. 
Rosch, E., Mervis, C. B., Gray, W. D., Johnson, D. M., \& Boyes-Braem, P. (1976). Basic objects in natural categories. Cognitive psychology, 8(3), 382-439.

Smith, T.J. (2012). The Attentional Theory of Cinematic Continuity. Projections: The Journal for Movies and Mind, 6(1), 1-27.

Smith, T.J. (2013). Watching You Watch Movies: Using Eye Tracking to Inform Cognitive Film Theory. In A. P. Shimamura (Ed.), Psychocinematics: Exploring Cognition at the Movies (165-191). New York: Oxford University Press.

Treisman, A. M., \& Riley, J. G. (1969). Is selective attention selective perception or selective response? A further test. Journal of Experimental Psychology, 79(1), 27-34.

Wallisch, $\mathrm{P}$ (2005). Affective responses to moving complex audio-visual stimuli are better predicted by "simple" response pools than by individual or pooled expert predictors. Poster presented at the annual meeting of the Midwestern Psychological Association, Chicago, IL.

Wallisch, P (2014). Appraisal of motion pictures is highly reliable intra- but not interindividually. Poster presented at the convention of the Association for Psychological Science, San Francisco, CA.

Wallisch, P., \& Whritner, J. A. (2017). Strikingly Low Agreement in the Appraisal of Motion Pictures. Projections: The Journal for Movies and Mind, 11(1), 102-120.

Williams, A. (1984). Is a radical genre criticism possible?. Quarterly Review of Film Studies, 9(2), 121-125. http://doi.org/10.1080/10509208409361197.

Wang, HX, Freeman F, Merriam EP, Hasson U, Heeger DJ. (2012). Temporal eye movement strategies during naturalistic viewing. Journal of Vision, 12(1), 1-27. 